dern auch den Investitionen eine bessere Finanzausstattung“, macht der Vorstandsvorsitzende deutlich. Diese seien zwar erhöht worden, lägen aber immer noch deutlich unter dem notwendigen Niveau.

\section{Zukunft der Reha-Einrichtungen steht auf dem Spiel}

Auch die wirtschaftliche Situation der Reha-Einrichtungen ist nach wie vor problematisch. 43,6\% der Reha-Einrichtungen schrieben in 2015 rote Zahlen und nur 20,5\% konnten einen Gewinn verzeichnen.

„Die Reha-Einrichtungen leben von der Substanz", mahnt der Hauptgeschäftsführer der BWKG, Matthias Einwag. Denn neben der unzureichenden Finanzierung der Betriebskosten bereite auch die mangelnde Finanzierung der Investitionen große Probleme. 62,7\% der Reha-Einrichtungen stellen fest, dass mit den Pflegesätzen die Investitionskosten nicht finanziert werden können. „Hier steht die Zukunft der Reha-Einrichtungen auf dem Spiel.“

\section{Fachkraftmangel - ein riesiges Problem}

Bei den Pflegeeinrichtungen hat sich die wirtschaftliche Situation in den letzten Jahren etwas verbessert. Dennoch haben 32,2\% der Pflegeeinrichtungen das Jahr 2015 mit roten Zahlen abgeschlossen. Den Einrichtungen machen zurzeit vor allem die Personalknappheit sowie neuen Vorgaben des Landes zu schaffen.

82,9\% der Pflegeeinrichtungen sagen, dass die Besetzung von freiwerdenden Stellen in der Pflege schwierig oder eher schwierig ist - das ist der zweithöchste Wert seit Beginn der Umfragen. Auch bei den Pflegehilfskräften wird die Lage immer schwieriger: $46,1 \%$ der befragten Pflegeeinrichtungen sahen darin ein Problem. Das ist der höchste Wert seit Beginn der Indikator-Umfragen.

„Der Fachkräftemangel ist für die Pflegeeinrichtungen ein riesiges Problem. Wir müssen alles dafür tun, um die Attraktivität des Altenpflegeberufs zu steigern und mehr Menschen dafür zu gewinnen“, hebt Einwag hervor.

\title{
Gesundheit des Pflegepersonals ist entscheidend für Berufszufriedenheit
}

Der Deutsche Berufsverband für Pflegeberufe e. V. (DBfK) hat die ersten Ergebnisse seiner Online-Umfrage vorgestellt. Wie groß die Belastungen in der Pflege sind und dass es inzwischen zur Normalität gehört, geltendes Arbeitsrecht großzügig auszulegen, zeigen die Antworten.

Ein Gesundheitssystem ist immer nur so belastbar wie die Fachkräfte, die es tragen", macht DBfK-Präsidentin Prof. Christel Bienstein deutlich. „Wir brauchen gesundheitsfördernde Arbeitsumfelder."

Mehr Arbeit, weniger Personal: Letzteres wird in Deutschland bei beruflich Pflegenden seit Jahren sträflich vernachlässigt. Immer mehr Arbeit soll von immer weniger und geringer qualifiziertem Pflegepersonal bewältigt werden - und das bei stetig steigenden Qualitätsanforderungen. „Arbeitsrecht und Arbeitsschutzbestimmungen werden dabei auf Kosten der Beschäftigten großzügig ausgelegt."

Die Antworten der DBfK-Online-Umfrage ",Mein Recht auf Frei" zu Dienstplansicherheit und Pausen von 3.572 Pflegende in Kliniken, Heimen und der ambulanten Pflege machen das Ausmaß der kontinuierlichen Überlastung und zahlreiche Verstöße gegen geltendes Recht deutlich.

Kurzfristig Schichten übernehmen: In $65 \%$ der Einrichtungen sei wenig bis kein Einfluss auf die Erstellung des Dienstplans möglich. Weiter berichtet beispielsweise knapp die Hälfte der Befragten, dass sie ein- bis zweimal im Monat kurzfristig Schichten übernehmen müssen. Ein weiteres Drittel sei davon sogar drei- bis fünfmal pro Monat und weitere $9,1 \%$ quasi wöchentlich betroffen. Weit überwiegend $(80,8 \%)$ werde deshalb eingesprungen, da man „Druck fühle, das Team nicht hängen zu lassen".

Die Umfrage zeigt weiter, dass freie Tage und Urlaub häufig als kostenlose Rufbereitschaft betrachtet und die Mitarbeiterlnnen kurzfristig zum Einspringen aufgefordert oder sogar genötigt werden. Rund zwei Drittel aller Befragten emp- finden daher eine kurzfristige Übernahme von Schichten als belastend, weil Erholung fehlt $(67,6 \%)$, weil das Privatleben unplanbar sei $(66,7 \%)$, weil es ein Verzicht auf Frei $(63,0 \%)$ bedeute.

Arbeitsdruck statt Pause: Befragt nach der Pausenregelung gaben zwei Drittel der Befragten $(64,6 \%)$ an, nur Pause zu machen, wenn es der Arbeitsanfall zulasse. Das führt dazu, dass gesetzlich vorgeschriebene Pausen entfallen, weil der Arbeitsdruck keine Pause zulässt. Die Folgen einer solchen Personalpolitik sind seit Jahren nicht zu übersehen: hohe Ausfallquoten, lange Krankheitsdauer, steigender Anteil belastungsbedingter psychischer Erkrankungen, Berufsflucht und innere Kündigung, schlechtes Berufsimage und sinkende Berufsverweildauer vor allem der jungen Generation.

Das alles sind keine guten Voraussetzungen, um den steigenden Pflegefachpersonenmangel zu bewältigen. Wer mehr Attraktivität für die Pflegeberufe will, darf nicht gleichzeitig schlechte Arbeitsbedingungen tolerieren. Diese Verantwortung tragen Politiker, Aufsichtsbehörden und Arbeitgeber gleichermaßen.

www.dbfk.de 